\title{
The importance of epidemiologic studies on interactions of the estrogen receptor alpha $(\alpha)$ in the normal mammary tissue with steroid hormones and hormones of the igf system in breast cancer research
}

\section{Commentary \\ Breast cancer management can be accomplished through measures and combination of measures of primary prevention, secondary prevention, and treatment, in a very similar manner to most chronic illnesses. Primary prevention, as first line of defense, constitutes always a priority, as it helps ease the anxiety implied by the process of diagnosis. With respect to breast cancer, progress of primary prevention efforts has been limited, since the incidence of the disease in most countries worldwide does not show any significant downward trends, even when the impact of gradually expanded application of screening methods has been taken into account.}

Primary prevention of breast cancer presupposes an understanding of its etiology, or at least its reversible risk factors. Breast cancer is principally considered a hormone dependent cancer. The liability of estrogen and testosterone, as well as of growth factor IGF-1 has been well documented. However, there is no definitive answer as to whether there is a synergistic interaction of these risk factors, and to what extent these hormonal factors influence the incidence of breast cancer independently or through the development of benign breast diseases.

The expression of estrogen receptors per se is a prerequisite for the expression of progesterone receptors. It is considered to be among the most valuable prognostic markers in breast cancer disease and its correspondence to endocrine treatment, along with PR, Her2-neu, and c-erb-2. Estrogen receptor alpha expression in the nonmalignant tissue has been shown to increase the risk of breast cancer by amplifying the influence of circulating steroids. ${ }^{1,2}$ It has been also suggested that the role of estrogen receptors alpha lies in augmenting the susceptibility of the epithelium to the abundant estrogen, and thus there is an increased risk of the disease among women who over express estrogen receptors in their normal mammary epithelium. ${ }^{3}$

It is not well known whether and how the expression of estrogen receptors and progesterone receptors in the normal mammary tissue affects the relationship between estrogens, testosterone, IGF-1 and benign breast disease or breast cancer. However, there is evidenced interaction of estrogens, IGF-1, and their receptors that provides biological plausibility for future and well comprehensive studies. ${ }^{4}$

Cells at risk or at intermediate stages of transformation may be more or less responsive to the growth enhancing influences of estrogens and other mammotropic hormones, depending on the density of the respective receptors in the nonmalignant tissue .5 . Recent work has provided some evidence that the expression of ER-a may play a role in the terminal differentiation of the mammary gland, and thus could regulate carcinogenic activity. ${ }^{3}$
Volume 5 Issue 5- 2016

Pantelina Zourna

Department of Hygiene, University of Athens, Greece

Correspondence: Pantelina Zourna MD, MPH, PhD, Department of Hygiene, University of Athens, Greece, Email Izourna@gmail.com

Received: August 24,20I6 | Published: September 09, 2016

Additionally, the implications of the expression of Estrogen receptors alpha, along with the action of steroid hormones and IGFlin benign breast disease (BBD) sounds intuitively like the sort of question we ought to answer onwards. After having compared patients with benign breast disease to healthy controls with respect to levels of endogenous estrogens, the evidence is again inconclusive. Similarly when comparing patients with breast cancer to patients with benign breast disease some studies find increased levels of steroids in benign breast disease while others have not reached that conclusion. In future studies, we should always keep in mind that premenopausal women with atypical ductal hyperplasia have a substantially higher risk than postmenopausal women with that diagnosis. ${ }^{6}$ Hence, this type of breast benign disease is considered- indirectly though- estrogen dependent. Recent studies have suggested that the expression of the ER alpha in the healthy mammary gland could dynamically alter the consequences of the actions of the steroid hormones, but also the actions of IGF-1, in respect to progression to benign breast disease or breast cancer. ${ }^{7}$

The positive associations between steroids and breast cancer have been established in many previous studies either in premenopausal or postmenopausal women. Results from meta-analyses suggest that steroid influences on mammary gland act presumably to a more considerable extent to the pathogenesis of benign breast diseases rather than breast cancer. ${ }^{8-10}$ Moreover, in a case-control study recently, the differential associations of the studied steroid hormones with BBD and cancer of the breast appear to be driven by associations noted among women with normal mammary tissue estrogen receptor- $\alpha$ positive. ${ }^{11}$

In line with the fact that the expression of estrogen receptors play an important role in the IGF-1 dependent growth of mammary cancer cells, the investigation of their interactions is perhaps the best measure in terms of assessing the impact of joint exposure. Also, IGF1 is positively associated with increased risk of Estrogen receptor alpha (+) breast cancer among premenopausal and postmenopausal women. This association is possibly explained by an effect of IGF-1 
on cell proliferation and apoptosis albeit it is unknown on what stage of carcinogenesis the effect is encountered. Another important gap of the evidence so far is whether IGF-1 has the ability to act on healthy mammary tissue, which either contains estrogen receptors or not. A recent study of case control design shows that the expression of ER alpha is necessary for IGF-1 to act on the process of carcinogenesis, in ER alpha positive patients. ${ }^{7}$

Estrogen receptor alpha and progesterone receptor expression in cancerous mammary tissue has been extensively investigated and thus the results of the research are applied into therapeutic or prognostic management. However, the expression of the same receptors in the healthy mammary gland, adjacent to pathological tissue, and its consequences constitute a recent research focus without any conclusive results so far. ${ }^{1-3}$ It is within the above-pathobiological and prognostic- context that the investigation of these relationships through large epidemiologic studies seems so important.

\section{Acknowledgments}

None.

\section{Conflicts of interest}

The author declares no conflicts of interest.

\section{Funding}

None.

\section{References}

1. Khan SA, Rogers MA, Khurana KK, et al. Estrogen receptor expression in benign breast epithelium and breast cancer risk. J Natl Cancer Inst. 1998;90(1):37-42.
2. Lawson JS, Field AS, Tran DD, et al. Breast cancer incidence and estrogen receptor alpha in normal mammary tissue-an epidemiologic study among Japanese women in Japan and Hawaii. Int $J$ Cancer. 2002;97(5):685-687.

3. Lagiou P, Georgila C, Samoli E, et al. Estrogen alpha and progesterone receptor expression in the normal mammary epithelium in relation to breast cancer risk. Int J Cancer. 2009;124(2):440-442.

4. Yee D, Lee AV. Crosstalk between the insulin like growth factors and estrogens in breast cancer. J Mammary gland Biol Neoplasia. 2000;5(1):107-115.

5. Trichopoulos D. Intrauterine environment, mammary gland mass, and breast cancer risk. Breast Cancer Res. 2003;5(1):42-44.

6. Guray M, Sahin AA. Review - Benign breast diseases:classification, diagnosis, and management. Oncologist. 2006;11(5):435-449.

7. Samoli E, Lagiou A, Zourna P, et al. Expression of estrogen receptors in non-malignant mammary tissue modifies the association between insulin-like growth factor 1 and breast cancer risk. Ann Oncol. 2015;26(4):793-797.

8. Eliassen AH, Missmer SA, Tworoger SS, et al. Endogenous steroid hormone concentrations and risk of breast cancer among premenopausal women. J Natl Cancer Inst. 2006;98(19):1406-1415.

9. Kaaks, Berrino F, Key T, et al. Serum sex steroids in premenopausal women and breast cancer risk within the European Prospective Investigation into Cancer and Nutrition (EPIC). J Natl Cancer Inst. 2005;97(10):755-765.

10. Kaaks R, Rinaldi S, Key TJ, et al. (2005) Postmenopausal serum androgens, oestrogens, and breast cancer risk. Endocr Relat Cancer. 2005;12(4):1071-1082.

11. Samoli E, Trichopoulos D, Lagiou A, et al. The hormonal profile of benign breast disease. Br J Cancer. 2013;108(1):199-204. 